\title{
TREATMENT FOR ANOREXIA NERVOSA: ARE WE MISSING THE MARK?
}

\author{
Peta Stapleton and Amy Bannatyne
}

School of Psychology, Bond University, Gold Coast, Queensland, 4229, Australia

Eating Disorders (EDs) are serious psychological conditions where attitudes toward food, weight and body size or shape become distorted and severe disturbances in eating or exercise behaviours often occur (Fairburn and Harrison, 2003). In a categorical sense, EDs can be divided into four broad groups: Anorexia Nervosa (AN), Bulimia Nervosa (BN), Binge Eating Disorder (BED) and Other Specified Feeding and Eating Disorders (OSFED, previously EDNOS) (Diagnostic and Statistical Manual of Mental Disorders, Fifth Edition, 2013). However, these categories are not discrete and it is not uncommon for sufferers to experience a spectrum of symptoms or crossover between diagnostic criteria. In the many years of ED research, if there is anything the scientific and clinical community have learned, it is that EDs are incredibly complex and multifaceted, with no "one-size fits all" solution (Strober and Johnson, 2012). For the purpose of the current editorial, the authors have focused on $\mathrm{AN}$, as the evidence and efficacy for treatment approaches remains limited.

$\mathrm{AN}$ is an EDwith a reported lifetime prevalence of 0.3 to $1.5 \%$ in women and 0.1 to $0.5 \%$ in men (Hudson et al., 2007); however, given the secretive nature of the illness, it is highly likely these prevalence rates are underestimated (Hoek and vanHoeken, 2003). In terms of severity, AN meets all the criteria typically associated with diseases or illnesses perceived as "severe", including prevalence, mortality, chronicity, functional impact, family dysfunction and societal effects (Touyz, 2011). AN is a chronic illness, with an average duration of five to seven years and the potential for life-long struggle (Ben-Tovim et al., 2001). Longitudinal follow-up studies of more than 20 years have revealed mortality rates of approximately $20 \%$ (Steinhausen, 2002), the highest of any mental illness (Birmingham et al., 2005).
Despite the suggestion in popular media, AN does not appear to be a modern phenomena, with origins dating back to the $13^{\text {th }}$ century during the time of 'holy anorexia' (Pearce, 2004). However, regardless of advances in modern medical intervention progressing well beyond expectations for many physical and psychological conditions, there continues to be insufficient data to make strong evidence-bas ed recommendations regarding the treatment of adults with AN (APA, 2013; 2014; Watson and Bulik, 2013). Advances have been made in the treatment of adolescents (with a relatively short duration of illness), with three recommendations currently offered: FamilyBased Treatment (FBT), outpatient services and inpatient services that combine refeeding and psychosocial interventions (NICE, 2004).

An enhancedversion of Cognitive Behavioural Therapy (CBT-E), offered as an outpatient service and designed to affect ED psychopathology and external obstacles to change, proposes EDs share the same psychopathology or 'transdiagnostic' mechanisms (Fairburn et al., 2009). Based on this transdiagnostic model, it is assumed treatment of BN should be identical and as effective as for AN. However, whilst the efficacy of CBT-E has been substantiated in research trials with BN and EDNOS, only "modest" evidence exists for CBT with AN (Fairburn and Harrison, 2003). Certain researchers argue the suggestion $\mathrm{BN}$ and $\mathrm{AN}$ share common core psychopathology is misleading. For example, while patients with $\mathrm{AN}$ and $\mathrm{BN}$ both experience increased salience to weight and shape concerns, are preoccupied with food, attribute low self esteem to aesthetic features and display a tendency to overestimate their body size; the two conditions differ in intensity of symptoms, not the quality (or form) of the psychological state (Beumont and Touyz, 2003). 
In $\mathrm{BN}$, it is argued these qualities or concerns are quintessence of the condition. For the BN sufferer, slenderness is desired to be 'happy' and 'healthy' and although he/she might be self-deprecating, this is due to associated behaviours, but is typically not the cause of the condition (Butow et al., 1993). For patients with AN, similar reasoning might occur in early stages; however, these cognitions are largely exchanged for more grotesque views once the disorder is fully established. As the illness progresses, the "reasons" underlying the disorder and associated behaviours become less clear and helplessness ensues. Not eating is perceived as deserved and warranted (i.e., an acceptable punishment) and emaciation serves as the primary outcome, rather than weight loss as a means of emotional equilibrium. Changes in psychiatric presentations are also seen and these disturbances are often inadequately explained by an "induced mental state" or a motivated pursuit of thinness (Beumont and Touyz, 2003).

Unlike patients with $\mathrm{BN}$, who are typically distressed by their ED and strongly desire cessation of associated behaviours (resulting in a high percentage of selfreferrals), starvation makes it increasingly challenging for individuals with AN to evaluate their condition rationally, or shift to alternative thoughts and/orbehaviours (Goldner, 1989), often resulting in poor motivation and treatment outcomes. Unfortunately, individuals who fail to respond to conventional or standard treatment approaches are often labeled as persistent, chronic, or "difficult to treat" (Federici and Wisniewski, 2013) and may be even less likely to receive the treatment and support they require and deserve.

The challenges of treating AN are plenty and welldocumented, including low prevalence of treatment seeking and high drop-out rates (Bulik, 2013), making it difficult for researchers to conduct Randomised Controlled Trials (RCTs) to determine the efficacy of various interventions. In addition to this, the complexity of EDs is often intensified by comorbidities including anxiety,depression, post-traumatic stress disorder and personality disorders (Bruce and Steiger, 2005). When practitioners focus exclusively on ED symptomatology, treatment typically fails to achieve therapeutic goals, as intervention effectiveness is often reduced bybehaviours associated with comorbid disorders (Federici and Wisniewski, 2013). In many instances, this can leave sufferers and clinicians exhausted and exasperated, raising concerns about what "recovery" actually looks like and whether "full recovery" (however it is defined) is possible for all sufferers.
In recent years, research has called for novel intervention approaches, particularly those targeting core biological and cognitive features, given the increasingly tendency to view AN from gene-centric viewpoint. Whilst genetics and the environment are thought to play important roles in the development (and possibly maintenance) of the condition, the exact influence of genes to environment varies considerably fromindividual to individual (Bulik et al., 2007). Clearly, this creates significant difficulty at a research level; however, it also complicates treatment and possibly explains why sufferers respond differently to various treatments. This difficulty has also led to investigation of potential endophenotypes and diagnostic overlap with other psychological disorders (e.g., ASD, OCD, anxiety and affective disorders for AN) (Lopez et al., 2009). It has been suggested these endophenotypes can be used to inform treatment strategies (i.e., targeting vulnerabilities), possibly improving treatment outcomes. However, research in the area is preliminary and further testing in randomised clinical trials with larger samples is required.

Another issue researchers and practitioners appear to agree on is that no solid or operational definition of recovery currently exists (Couturier and Lock, 2006) and many definitions appear to focus heavily on the physical dimensions, leaving social and emotional components unaccounted for (Lowe, 2001). It seems recovery in a traditional sense (i.e., returning to premorbid or 'normal' functioning) may be an overly simplistic solution for EDs (Dawson et al., 2014b). For some individuals (particularly those with severe and enduring EDs), it has been suggested the disorder may infiltrate and entwine inextricably with an individual's sense of self and identity, potentially complicating treatment of an already multifaceted and complex illness (Dawson et al., 2014a).

In recent years, this has led to a recommended shift in treatment models, which focus on improving quality of life and harm minimization, particularly in cases of severe and enduring $\mathrm{AN}$, as opposed to recovery equating to "symptom free" (Touyz et al., 2013; Williams et al., 2010). However, one must consider when does this recovery model become problematic? Are we really trying hard enough? Is it morally and/or ethically appropriate to "force" recovery/refeeding in clients who are young and might have a strong chance of making a "full" recovery? At the same time, is the utopian idea of a symptom-free, full (or 'perfect') recovery, demoralising and unattainable, leading to poor self-efficacy and a lack of hope? 
While the continuum between severe pathology (or mortality) in AN and complete recovery is clear, the many stagesin between carry real implications for health and well-being; however, the desire to recover in no way ensures success. Change is likely to manifest in a nonlinear pattern, with recurrent fluctuations between a motivation to advance and desire to retreat (Vitousek et al., 1998). False starts and retrenchments are common and treatment intentions should be formulated as "experiments" of new ways of living and being, rather than finite solutions. Setbacks are inherent and should be expected, yet should be considered an opportunity to gather information to support renewed efforts, rather than a failure or contraindication for future outcomes (Vitousek et al., 1998). Expressions of interest to explore alternatebehaviours must be immediately and frequently put into practice, rather than discussed via abstraction, as anticipation of change in $\mathrm{AN}$ is often more distressing than the reality of change itself (Garner et al., 1997).

Yet, clinicians must also consider and acknowledge the sorrow and purposelessness many AN sufferers experience following the extinction of an identity (or way of life) that had become so familiar and comforting, thereby shifting the aim of therapy from increasing motivating to change, to fostering new skills and strengths to compensate for the consequences of change and new beginnings (Vitousek et al., 1998). Without helping sufferers become the best version of themselves in any treatment model, there is a grave risk novel treatments will inevitably continue to fall short.

More importantly, maybe we need to focus on making treatment accessible and removing some of the social, financial and systemic barriers that prevent early intervention in the first place. If we can transplant vital organs with remote telemanipulators and use stem cells to generate structures that mimic the human brain, surely we can, at the bare minimum, provide this underserved population with access to equitable and sufficient care, whilst the search for optimal treatment continues using innovative approaches such as epigenetics and the like (Bulik, 2013). Though discovery also entails uncertainty and challenge and reflecting back over the years of ED literature, we know there is no simple solution to these heterogeneous and highly complex disorders. That said, "when the going gets tough, the tough gets going".

The body of literature surrounding treatment of $\mathrm{AN}$ is progressing, albeit with on-going difficulty. To ensure the provision of effective and relevant clinical practice, clinicians and researchers may be best servedby embracing an approach that incorporates the current body of treatment literature, clinical practice guidelines (even practice areas RCTs have not highlighted) and emerging treatment evidence (Watson and Bulik, 2013). To continue advancing our knowledge and understanding of best practice treatment in $\mathrm{AN}$, future research must address methodological issues of previous RCTs (e.g., drop-out and treatment non-compliance), embrace emerging theoretical approaches (e.g., socioemotional, neurocognitive), adapt current therapeutic approaches to specific subgroups and contexts (e.g., adult-based family therapy, couple-based interventions, broadening of FBT from family of 'origin' to family of 'choice'), strive for replication, remain open to novel and innovative approaches (e.g., epigenetics) and endeavor to improve early illness identification (Watson and Bulik, 2013). Ultimately, it is hoped we can create a rich collection of psychotherapies, developmentally tailored to meet the full spectrum of treatment needs and complexities of individuals suffering from AN.

\section{REFERENCES}

APA, 2013. Diagnostic and Statistical Manual of Mental Disorders, 5th Edn., Arlington, VA: American Psychiatric Publishing.

APA, 2014. Anorexia nervosa. www.psychologicaltreatments.org

Ben-Tovim, D., K. Walker, P. Gilchrist, R. Freeman and R. Kalucy et al., 2001. Outcome in patients with eating disorders: A 5-year study. Lancet, 357: 12541257. DOI: 10.1016/S0140-6736(00)04406-8

Beumont, P.J.V. and S. Touyz, 2003. What kind of illness is anorexia nervosa? Eur. Child Adolescent Psychiatry, 12: 20-24. DOI: 10.1007/s00787-003-1103-y

Birmingham, C., J. Su, J. Hlynsky, E. Goldner and M. Gao, 2005. The mortality rate from anorexia nervosa. Int. J. Eating Disorders, 38: 143-146. DOI: 10.1002/eat.20164

Bruce, K.R. and H. Steiger, 2005. Treatment implications of axis-II comorbidity in eating disorders. Eating Disorders: J. Treatment Prevention, 13: 93-108. DOI: 10.1080/10640260590893700

Bulik, C., M. Slof-Op'tLandt, E. Van Furth and P. Sullivan, 2007. The genetics of anorexia nervosa. Ann. Rev. Nutrition, 27: 263-275. DOI: 10.1146/annurev.nutr.27.061406.093713

Bulik, C.M., 2013. The challenges of treating anorexia nervosa. Lancet, 383: 105-106. DOI: 10.1016/S0140-6736(13)61912-1

Butow, P.N., P.J.V. Beumont and S. Touyz, 1993. Cognitive processes in eating disorders. Int. J. Eating Disorders, 14: 319-329. 
Couturier, J. and J. Lock, 2006. What is recovery in adolescent anorexia nervosa? Int. J. Eating Disorders, 39: 550-555. DOI: 10.1002/eat.20309

Dawson, L., P. Rhodes and S. Touyz, 2014a. Doing the impossible: The process of recovery from chronic anorexia nervosa. Qualitative Health Res., 24: 494505. DOI: $10.1177 / 1049732314524029$

Dawson, L., P. Rhodes and S. Touyz, 2014b. The recovery model and anorexia nervosa. Aus. New Zealand J. Psychiatry, DOI: 10.1177/000486741

Fairburn, C. and P.J. Harrison, 2003. Eating disorders. Lancet, 361: 407-416. DOI: 10.1016/S01406736(03)12378-1

Fairburn, C., Z. Cooper, H. Doll, M. O'Connor and K. Bohn et al., 2009. Transdiagnostic cognitivebehavioral therapy for patients with eating disorders: A two-site trial with 60-week follow-up. Am. J. Psychiatry, 166: 311-319.

Federici, A. and L. Wisniewski, 2013. An intensive DBT program for patients with multidiagnostic eating disorder presentations: A case series analysis. Int. J. Eating Disorders, 46: 322-331.

Garner, D.M., K. Vitousek and K.M. Pike, 1997. Cognitive Behavioral Therapy for Anorexia Nervosa. In: Garner, D.M. and P.E. Garfinkel (Eds.), Handbook of Treatment for Eating Disorders, New York: Guilford Press, pp: 91-144.

Goldner, E., 1989. Treatment refusal in anorexia nervosa. Int. J. Eating Disorders, 8: 297-306. DOI: 10.1002/1098-108X

Hoek, H.W. and D. Van Hoeken, 2003. Review of the prevalence and incidence of eating disorders. Int. J. Eating Disorders, 34: 383-396. DOI: 10.1002/eat. 10222

Hudson, J., E. Hiripi and H. Pope Jr. and R. Kessler, 2007. The prevalence and correlates of eating disorders in the national comorbidity survey replication. Biological Psychiatry, 61: 348-358. DOI: 10.1016/j.biopsych.2006.03.040

Lopez, C., M. Roberts and J. Treasure, 2009. Biomarkers and Endophenotypes in Eating Disorders. In: The Handbook of Neuropsychiatric Biomarkers, Endophenotypes and Genes, M.S. Ritsner (Ed.), Netherlands: Springer, pp: 227-237.
Lowe, J.B., 2001. Glycosylation, Immunity and Autoimmunity. Cell, 104: 809-812. DOI: 10.1016/S0092-8674(01)00277-X

NICE, 2004. Eating disorders: Core interventions in the treatment and management of anorexia nervosa, bulimia nervosa and related eating disorders: National Institute for Clinical Excellence.

Pearce, J.M., 2004. Richard Morton: Origins of anorexia nervosa. European Neurology, 52: 191-192.

Steinhausen, H.C., 2002. The outcome of anorexia nervosa in the 20th century. Am. J. Psychiatry, 159: 1284-1293. DOI: 10.1176/appi.ajp.159.8.1284

Strober, M. and C. Johnson, 2012. The need for complex ideas in anorexia nervosa: Why biology, environment and psyche all matter, why therapists make mistakes and why clinical benchmarks are needed for managing weight correction. Int. J. Eating Disorders, 45: 155-178. DOI: 10.1002/eat.22005

Touyz, S., 2011. What kind of illness is anorexia nervosa? A clinical update. Bulletin Australian Psychological Society, 33: 13-15.

Touyz, S., D. Le Grange, H. Lacey, P. Hay and R. Smith et al., 2013. Treating severe and enduring anorexia nervosa: A randomized controlled trial. Psychological Medicine, 43: 2501-2511. DOI: 10.1017/S0033291713000949

Vitousek, K., S. Watson and G. Wilson, 1998. Enhancing motivation for change in treatment resistant eating disorders. Clinical Psychology Rev., 18: 391-420. DOI: $10.1016 / \mathrm{S} 0272-$ 7358\%2898\%2900012-9

Watson, H.J. and C.M. Bulik, 2013. Update on the treatment of anorexia nervosa: Review of clinical trials, practice guidelines and emerging intervention. Psychological Med., 43: 2477-2500. DOI: 10.1017/S0033291712002620

Williams, K.D., T. Dobney and J. Geller, 2010. Setting the eating disorder aside: An alternative model of care. Eur. Eating Disorders Rev., 18: 90-96. DOI: 10.1002/erv.989 